\title{
Reacciones adversas vinculadas a la vacuna SINOPHARM en personal de salud del Hospital Regional
}

\section{Policial Arequipa Julio Pinto Manrique}

Adverse reactions linked to the SINOPHARM vaccine in health personnel of the Regional Police Hospital Arequipa Julio

$$
\text { Pinto Manrique }
$$

Reações adversas ligadas à vacina SINOPHARM em profissionais de saúde do Hospital Regional de Polícia de Arequipa Julio Pinto Manrique

\section{ARTÍCULO GENERAL}

\author{
Susana Mercedes Guillén Pinto \\ susanaguillen689@hotmail.com
}

Hospital Regional Policial Arequipa Julio Pinto Manrique, Arequipa, Perú

Recibido 01 de Diciembre 2021 | Arbitrado y aceptado 01 de Diciembre 2021 | Publicado en 24 Enero 2022

\section{RESUMEN}

Objetivo: Fue determinar las reacciones adversas vinculadas a la vacuna SINOPHARM en el personal de salud del Hospital Regional Policial "Julio Pinto Maßrique" de Arequipa. Método: Se trato de una investigación no experimental, básica, transversal y prospectiva, la población estuvo conformada por el personal de salud que labora en el hospital antes mencionado, se cargó en una plataforma virtual el formulario de preguntas utilizado por los Centros para el control y prevención de enfermedades (CDC), contestando un total de 162 personas.

Resultados: El personal de salud en ambas dosis presentó mayormente efectos secundarios como dolor de cabeza y dolor, hinchazón, enrojecimiento en la zona de punción de la vacuna, y reacciones alérgicas como mareos y taquicardia.

Conclusión: Los efectos secundarios se presentaron en la 1ra dosis en $66.73 \%$ en la 2 da dosis en $58.64 \%$; las reacciones alérgicas en la 1ra dosis se presentó en $8.02 \%$ y en la $2 \mathrm{da}$ en $5.56 \%$ en el personal de salud del mencionado Hospital. Palabras Clave: Reacciones adversas, vacuna SHINOPHARM

\section{ABSTRACT}

Objective: It was to determine the adverse reactions linked to the SINOPHARM vaccine in the health personnel of the Regional Police Hospital "Julio Pinto Manrique" of Arequipa.

Method: It was a non-experimental, basic, cross-sectional and prospective investigation, the population was made up of the health personnel who work in the aforementioned hospital, the question form used by the Centers for control was loaded on a virtual platform. and disease prevention (CDC), answering a total of 162 people.

Results: The health personnel in both doses presented mostly side effects such as headache and pain, swelling, redness in the area where the vaccine was punctured, and allergic reactions such as dizziness and tachycardia.

Conclusion: Side effects occurred in the 1 st dose in $66.73 \%$ in the 2 nd dose in $58.64 \%$; allergic reactions in the $1 \mathrm{st}$ dose occurred in $8.02 \%$ and in the 2 nd in $5.56 \%$ in the health personnel of the aforementioned Hospital.

Keywords: Adverse reactions, SINOPHARM vaccine

\section{RESUMO}

Objetivo: Foi determinar as reações adversas relacionadas à vacina SINOPHARM no pessoal de saúde do Hospital Regional de Polícia "Julio Pinto Manrique" de Arequipa.

Método: Foi uma investigação não experimental, básica, transversal e prospectiva, a população foi composta pelos profissionais de saúde que trabalham no referido hospital, o formulário de perguntas utilizado pelos Centros de controle foi carregado em uma plataforma virtual. e prevenção de doenças (CDC), atendendo um total de 162 pessoas.

Resultados: O pessoal de saúde em ambas as doses apresentou principalmente efeitos colaterais como dor de cabeça e dor, inchaço, vermelhidão na área onde a vacina foi puncionada e reações alérgicas como tontura e taquicardia.

Conclusão: Efeitos colaterais ocorreram na $1^{\text {a }}$ dose em $66,73 \%$ na $2^{\mathrm{a}}$ dose em $58,64 \%$; as reações alérgicas na $1^{\text {a }}$ dose ocorreram em $8,02 \%$ e na $2^{\mathrm{a}}$ em $5,56 \%$ no pessoal de saúde do referido Hospital.

Palavras-chave: Reações adversas, vacina SINOPHARM 


\section{INTRODUCCIÓN}

En diciembre del 2019 en Wuhan se dio a conocer sobre una neumonía grave que se presentó en 27 personas $(1,2)$, se dirigieron los estudios hacia los virus de la gripo aviar, SARS (Síndrome respiratorio agudo severo) y MERS (Síndrome respiratorio del medio Oriente), siendo los resultados negativos para está neumonía. En enero del 2020 se pudo determinar que el agente causal era un virus nuevo de la familia Coronavirus, denominado inicialmente 2019-nCOV $(1,3,4,5)$.

La enfermedad conocida como COVID-19, se expandió rápidamente hacia otras regiones de China, Tailandia, Corea del Sur, países de Europa y América, por lo que en marzo del 2020 la OMS declaro pandemia mundial por COVID-19 $(6,7,8,9,1,3,10)$.

Es así como, los laboratorios inician una carrera en búsqueda de una vacuna en contra del SARS-COV-2, crear y producir una vacuna es todo un proceso de muchos años de duración, desde el descubrimiento del agente causal hasta la producción y salida al mercado. Debido a la expansión rápida de la enfermedad y por lo que ha generado en la economía, salud y educación de la población en todos los países del mundo, es que el proceso normal en el desarrollo de una vacuna se ha acelerado, reduciéndose los tiempos de años a meses (11), realizándose en forma paralela las fases de los experimentos, manteniéndose estrictas normas clínicas y de seguridad (12).

En menos de un año se identificó el virus a inicio del 2020 (13), al poco tiempo se secuenció su genoma, y en la actualidad se tiene vacunas en evaluación en seres humanos. La OMS publicó el 28 de setiembre del 2020 que había 40 vacunas en ensayos clínicos en seres humanos, 10 de las cuales se hallaban en fase III con el fin de evaluar su eficacia y seguridad (11), además de existir 151 vacunas en desarrollo en fase preclínica, incluyendo las desarrolladas en el Perú.

Frente a la magnitud de la pandemia, es que la OMS y la FDA por emergencia aprobaron el uso de vacunas sin que estas hubiesen terminado sus ensayos clínicos, si bien es cierto que las vacunas son una esperanza para controlar y/o terminar con la pandemia, de estas no se han investigado a profundidad los posibles efectos adversos que pudieran producirse después de su inyección.

La vacuna SINOPHARM, vacuna SARS-COVID-2 (células vero) inactiva producida por el Laboratorio del Instituto de Productos Biológicos de Beijing, de la 
República Popular de China, es una vacuna monovalente compuesta por antígenos del virus SARS-COVID-2 inactivo. Se realizaron ensayos clínicos de Fase I y II randomizado, doble ciego, con placebo, en estas fases se evaluaron dosis, esquemas e intervalos. La seroconversión de anticuerpos neutralizantes fue de 99,25\% con un título medio geométrico (GMT) de 156, después de 14 días de la segunda dosis. En ensayos clínicos fase III se evaluó la neutralización cruzada contra diez cepas prevalentes o típicas del SARS-COV-2 a nivel internacional, evidenciaron que estos podían neutralizar eficazmente todas las cepas. La eficacia de esta vacuna según ensayos preliminares Fase III es de 79,34\% (IC 95\%: 59,17\% - 89,55\%) (14). En el mes de mayo del 2021 la OMS aprobó el uso por emergencia de esta vacuna, señalando que habían validado la "seguridad, eficacia y calidad" de la vacuna SINOPHARM.

En estos meses, la vacuna SINOPHARM ha sido muy cuestionada en Perú, respecto a su eficacia y efectividad contra el virus SARS-COVID-19, debido a los pocos datos publicado en revistas médicas internacionales, sin embargo, se la viene aplicando desde el mes de febrero-marzo del 2020. Una vez que se inicia la administración de una vacuna, las autoridades sanitarias y la OMS se mantienen en vigilancia con el fin de registrar y determinar la gravedad de las presuntas reacciones adversas y constatar la reacción de las personas a las que se les ha administrado la vacuna. Es primordial garantizar que la vacuna sea segura, por lo que se deben realizar periódicamente evaluaciones y estudios clínicos con el fin de obtener información actualizada sobre su eficacia. Debido a lo antes mencionado la importancia de realizar el presente estudio de prevalencia sobre los posibles efectos adversos de la vacuna SINOPHARM, siendo la falta de conocimiento al respecto lo que ha motivado la realización de este estudio. A medida que se obtenga más información, se podrá contar con una mayor data que permita ampliar el conocimiento e incentive a la población a no temer a la vacunación.

El objetivo principal de la presente investigación fue determinar la frecuencia de presentación de reacciones adversas vinculadas a la vacuna SINOPHARM en el personal de salud del Hospital Regional Policial Arequipa "Julio Pinto Manrique". Sus objetivos específicos fueron: 1. Determinar la frecuencia de efectos secundarios vinculados a la vacuna SINOPHARM en el personal de salud del Hospital Regional Policial “Julio Pinto Manrique". 2. Determinar la frecuencia de reacciones alérgicas vinculadas a la vacuna SINOPHARM en el personal de salud del Hospital Regional Policial Arequipa "Julio Pinto Manrique". 


\section{MATERIAL Y MÉTODOS}

Se trato de una investigación de tipo transversal, descriptivo, de diseño no experimental, de variable única: reacciones adversas vinculadas a la vacuna SHINOPHARM, conceptualizada como la respuesta nociva no deseada y no intencionada que se produce luego de la administración de un fármaco; cuya definición operativa fue a través de los efectos secundarios y reacciones alérgicas.

La población de estudio estuvo conformada por el personal de salud que labora en el Hospital Regional Policial de Arequipa "Julio Pinto Manrique", pertenecientes a los grupos profesionales: médicos, enfermeras, obstetras, biólogos, químicos farmacéuticos, Odontólogos, auxiliares de enfermería y administrativos, la muestra estuvo conformada por 162 unidades de estudio que accedieron a participar en la investigación, cuyo promedio de edad fue de 46,94 años, de ambos géneros, 22 varones y 140 mujeres, conformando un solo grupo de estudio ya que no se tuvo como objetivo comparar entre géneros.

Se aplicó un formulario de preguntas en forma virtual, previa autorización del director general PNP, asignando el carácter obligatorio la participación en el estudio. El instrumento aplicado fue el que se utiliza en el Sistema de Reportes de Eventos Adversos de vacuna de la FDA y CDC.

\section{III.RESULTADOS}

Tabla 1

\section{Efectos secundarios vinculados a la vacuna SINOPHARM}

\begin{tabular}{lllll}
\hline \multirow{2}{*}{ Efectos secundarios } & \multicolumn{2}{l}{1 ra dosis } & \multicolumn{2}{l}{2 da dosis } \\
& $\mathrm{N}^{\mathrm{o}}$ & $\%$ & $\mathrm{~N}^{\mathrm{o}}$ & $\%$ \\
\hline Dolor, hinchazón enrojecimiento & 30 & 18.52 & 30 & 18.52 \\
Cansancio & 28 & 17.28 & 22 & 13.58 \\
Dolor de cabeza & 48 & 29.62 & 35 & 21.60 \\
Dolor muscular & 27 & 16.66 & 22 & 13.58 \\
Escalofríos & 9 & 5.5 & 6 & 3.70 \\
Dolor articular & 6 & 3.70 & 9 & 5.55 \\
Fiebre & 3 & 1.85 & 4 & 2.47 \\
Náuseas & 11 & 6.79 & 8 & 4.94 \\
Sentirse mal & 6 & 3.70 & 5 & 3.09 \\
Ganglios inflamados & 2 & 1.23 & 5 & 3.09 \\
Otros & 1 & 0.62 & 12 & 7.41 \\
Presentaron & 100 & 61.73 & 95 & 58.64 \\
No presentaron & 62 & 38.27 & 67 & 41.35 \\
\hline
\end{tabular}


El personal de salud de la PNP que labora en el Hospital Regional Policial de Arequipa refirió haber presentado mayormente en ambas dosis dolor de cabeza y dolor, hinchazón, enrojecimiento en $18.52 \%$. Cansancio y dolor muscular los refirieron un $17.28 \%$ y $16.66 \%$ respectivamente en la primera dosis, mientras que en la segunda dosis los presentaron un $13.58 \%$.

En general un $61.73 \%$ del personal de salud al que se aplicó la 1ra dosis de la vacuna de SINOPHARM presentó algún efecto secundario y $38.27 \%$ no presentaron ninguno, mientras que en la 2 da dosis presentaron estos efectos un $58.64 \%$ y no los presentaron un $41.35 \%$.

Tabla 2

Reacciones alérgicas vinculadas a la vacuna SINOPHARM

\begin{tabular}{lcccc}
\hline \multirow{2}{*}{ Reacciones alérgicas } & \multicolumn{2}{c}{1 ra dosis } & \multicolumn{2}{c}{2 da dosis } \\
& $\mathrm{N}^{\mathrm{o}}$ & $\%$ & $\mathrm{~N}^{\mathrm{o}}$ & $\%$ \\
\hline Dificultad para respirar & 1 & 0.62 & 2 & 1.23 \\
Hinchazón cara & 3 & 1.85 & 2 & 1.23 \\
Taquicardia & 3 & 1.85 & 4 & 2.47 \\
Salpullido todo el cuerpo & 0 & 0 & 0 & 0 \\
Mareos y dificultad & 6 & 3.70 & 3 & 1.85 \\
Presentaron & 13 & 8.02 & 9 & 5.56 \\
no presentaron & 149 & 91.98 & 153 & 94.44 \\
\hline
\end{tabular}

Las reacciones alérgicas a la vacuna Sinopharm no se presentaron en $91.98 \%$ del personal de salud en la primera dosis y en $94.44 \%$ en la segunda dosis. Las reacciones adversas que refirieron fueron mareos y dificultad para ponerse derecho en $3.70 \% \mathrm{y}$ $1.85 \%$ en 1ra y 2 da dosis respectivamente, asimismo señalaron hinchazón de la cara, taquicardia y dificultad para respirar después de recibir ambas dosis, pero en porcentajes mínimos.

Tabla 3

Frecuencia de COVID-19

\begin{tabular}{ccc}
\hline COVID-19 & $\mathrm{N}^{\mathrm{o}}$ & $\%$ \\
\hline SI & 90 & 55.60 \\
NO & 72 & 44.40 \\
TOTAL & 162 & 100.00 \\
\hline
\end{tabular}


Tabla 4

Intensidad

\begin{tabular}{lll}
\hline Intensidad & $\mathbf{N}^{\mathbf{0}}$ & $\mathbf{\%}$ \\
\hline Leve & 30 & 33.34 \\
Moderado & 29 & 32.22 \\
Severo & 2 & 2.22 \\
Asintomático & 29 & 32.22 \\
TOTAL & 90 & 100.00 \\
\hline
\end{tabular}

Un 55.60\% del personal de salud del Hospital Regional Policial Arequipa, refirió haber presentado la enfermedad COVID-19 (Tabla 1), la intensidad de la enfermedad ha sido mayormente leve $33.34 \%$ y moderada $32.22 \%$ e igual que este último lo fueron los asintomáticos (Tabla 2).

\section{IV.DISCUSIÓN}

Las reacciones adversas vinculadas a la vacuna SINOPHARM, en la presente investigación se las clasificó en efectos secundarios y reacciones alérgicas en base al instrumento aplicado por las CDC (Centros para el Control y Prevención de enfermedades).

Los efectos secundarios referidos por el personal de salud del Hospital policial de Arequipa en sentido decreciente fueron dolor de cabeza, dolor, hinchazón y enrojecimiento, cansancio y dolor muscular mayormente en ambas dosis después de la inyección de la vacuna SINOPHARM.

La Americas TB Coalition (15), dió a conocer una lista completa de las reacciones adversas en base al Concejo de Organizaciones Internacionales de Ciencias Médicas, señalando al dolor de cabeza como muy común, al dolor, hinchazón y enrojecimiento en la zona de punción como más comunes, al cansancio y dolor muscular como comunes. En comparación al presente estudio, creo que no se puede hablar que el dolor de cabeza sea muy común ya que este se presentó en $29.62 \%$ personas en la 1ra dosis y en $21.60 \%$ en la $2 \mathrm{da}$, prácticamente en menos del 3cio de la población de estudio; el dolor, hinchazón y enrojecimiento lo refirieron $18,52 \%$ en ambas dosis, siendo no muy común respecto a la clasificación en referencia; el cansancio lo señalaron un $17.28 \%$ en la 1ra dosis y en $13.58 \%$ en la $2 \mathrm{da}$, el dolor muscular en $16.66 \%$ en $1 \mathrm{ra}$ dosis, en la $2 \mathrm{da}$ en 
$13.58 \%$, no concordando también con la clasificación mencionada, que las señala como comunes.

Asimismo, se comparó con otra clasificación, que hace uso de los porcentajes de presentación de los efectos adversos, que clasifica al dolor de cabeza y cansancio como frecuentes al presentarse entre $1-10 \%$, al dolor muscular como poco frecuente por presentarse en $<1 \%$ de las personas. Si tomamos en cuenta esta clasificación, se puede observar que los efectos secundarios referidos por el personal de salud de este Hospital, se han presentado en mayor porcentaje, no encajando en esta clasificación. Si, concuerda con la clasificación muy frecuente del dolor al presentarse en $>$ al 10\%, como ocurrió en este estudio.

Respecto a las reacciones adversas, en la investigación en orden decreciente el personal de salud señaló haber presentado mareos y dificultad para caminar en 3.07\% en la 1 ra dosis y en 1.85 en la 2 da dosis; taquicardia en $1.85 \%$ en 1 ra dosis y en $2.47 \%$ en la 2 da; hinchazón de la cara en la 1ra dosis en $1.85 \%$, en la 2 da en $1.23 \%$. La clasificación de Americas TB Coalition clasifica a los mareos como poco común, a la taquicardia como raro; la clasificación según los porcentajes, los mareos son poco frecuentes ya que se presentan en $>1 \%$, disnea es clasificada como frecuente al presentarse en $1-10 \%$ de la población. En general la Clasificación Américas TB Coalition señala a las reacciones alérgicas como raros, concordando con el estudio, ya que estas se presentaron en $8.02 \%$ en la 1 ra dosis y en $5.56 \%$ en la $2 \mathrm{da}$.

Se puede deducir, al comparar los resultados del estudio con estas clasificaciones, que existen diferencias, pero lo más importante a rescatar es que, en más del 50\% de la población de estudio se presentaron por lo menos un efecto secundario, que no causan daño permanente, ni alteran las capacidades de las personas; y que las reacciones alérgicas también se presentaron, pero en porcentajes muy bajo, aquí se tendría que considerar que quizás estas personas tendrían algún trastorno alergénico.

Los resultados obtenidos permitirán ampliar el conocimiento sobre la presentación de eventos adversos vinculados a la vacuna SINOPHARM de la comunidad, con el fin de minimizar las creencias que se tiene sobre la vacunación. Hay que destacar la necesidad e importancia en realizar este tipo de investigaciones, con el fin de tener más datos que avalen el uso de las vacunas.

Finalmente se debe considerar que, la respuesta inmunológica es individual en cada persona, y que los eventos adversos son comunes después de la inyección de 
cualquier vacuna. Inclusive se piensa que es mejor tener efectos adversos ya que los consideran como un signo de que el sistema inmunológico está actuando.

Se presentaron dos tablas que no responden a los objetivos, con el fin de conocer el porcentaje de la población de estudio que se enfermó de COVID, así como de la intensidad de la enfermedad, el personal de salud señaló en un 55.60\% haber tenido la enfermedad de intensidad leve a moderado en $33.34 \%$ y $32.22 \%$ respectivamente, y un importante $32.22 \%$ refirió haber sido asintomático, no concordando con lo que se menciona sobre los asintomáticos que lo son en mayoría.

\section{CONCLUSIONES}

Las reacciones adversas vinculadas a la vacuna SINOPHARM como, efectos secundarios en la $1 \mathrm{ra}$ dosis se presentaron en $61.73 \%$, y en la 2 da en $58.64 \%$; las reacciones alérgicas en la $1 \mathrm{ra}$ dosis en $8.02 \%$ y en la 2 da en $5.56 \%$ en el personal de salud del Hospital Regional Policial Arequipa.

Los efectos secundarios que se presentaron mayormente en ambas dosis fueron el dolor de cabeza, dolor, hinchazón y enrojecimiento en la zona de punción en el personal de salud del Hospital Regional Policial Arequipa.

Las reacciones adversas en la 1ra dosis fueron los mareos los de mayor prevalencia y la taquicardia en la 2 da dosis en el personal de salud del Hospital Regional Policial Arequipa.

\section{BIBLIOGRAFÍA}

Adhanom-Ghebreyesus T. WHO Director-General's opening remarks at the media briefing on COVID-19 - 11 March 2020. Ginebra, Suiza: World Health Organization. [Online]; 2020. Acceso 1 de juniode 2021. Disponible en: HYPERLINK "https://www.who.int/dg/speeches/detail/who-director-general-sopening-remarks-at-the-media-briefing-on-covid-19---11-march-2020"

https://www.who.int/dg/speeches/detail/who-director-general-s-opening-remarksat-the-media-briefing-on-covid-19---11-march-20

World Health Organization. Draft landscape of COVID-19 candidate vaccines. [Online]; 2020. Acceso 1 de juniode 2021. Disponible en: HYPERLINK 
Susana Mercedes Guillén Pinto

https://www.who.int/publications/m/item/draft-landscape-of-covid-19-candidatevaccines https://www.who.int/publications/m/item/draft-landscape-of-covid-19candidate-vaccines .

Organización Mundial de la Salud. Enfermedad por coronavirus (COVID-19): Investigación y desarrollo de vacunas. [Online]; 2020. Acceso 1 de juniode 2021. Disponible en: HYPERLINK "https:/www.who.int/es/news-room/q-adetail/coronavirus-disease-(covid-19)-vaccine-research-and-development" https://www.who.int/es/news-room/q-a-detail/coronavirus-disease-(covid-19)vaccine-research-and-development .

Zhu N, Zhang D, Wang W, Li X, Yang B, Song J, et al. novel coronavirus from patients with pneumonia in China. N Engl J Med. 2020; 382: p. 727-33.

Ministerio de Salud Argentina. Manual del Vacunador Vacuna SINOPHARM. [Online].; 2021. Acceso 1 de KIMOP de 2021. Disponible en: HYPERLINK "file://D:/USUARIO/Downloads/manual-vacunador-sinopharm_12-3-2021.pdf" file:///D:/USUARIO/Downloads/manual-vacunador-sinopharm_12-3-2021.pdf .

Americas TB Coalition. revista: Lista completa de reacciones adversas de la vacuna SINOPHARM. [Online]; 2021. Acceso 1 de juniode 2021. Disponible en: HYPERLINK "https://actbistas.org/la-lista-completa-de-reacciones-adversas-dela-vacuna-sinopharm-de-china/" https://actbistas.org/la-lista-completa-dereacciones-adversas-de-la-vacuna-sinopharm-de-china/Briggs H. Coronavirus: cómo se estrecha el cerco sobre el pangolín como probable transmisor del patógeno que causa el covid-19. Londres: BBC. 2020.

Guo Y, Cao Q, Hong Z, Tan Y, Chen S, Jin H, et al. he origin, transmission and clinical therapies on coronavirus disease 2019 (COVID-19) outbreak - an update on the status. Mil Med Res. 2020; 7(11).

$\mathrm{Lu} \mathrm{R}$, Zhao X, Li JNP, Yang B, Wu H, al e. Genomic characterisation and epidemiology of 2019 novel coronavirus: implications for virus origins and receptor binding. Lancet. 2020; 395: p. 565-574.

Ministerio de Sanidad. Actualización no 13. Neumonía por nuevo coronavirus (2019$\mathrm{nCoV}$ ) en Wuhan, provincia de Hubei, (China). Ministerio de Sanidad. 2020. 
Organización Mundial de la Salud. Alocución de apertura del Director General de la OMS en la rueda de prensa sobre la COVID-19 celebrada el 11 de marzo de 2020. Ginebra. Organización Mundial de la Salud. 2020.

Organización Mundial de la Salud. Cronología de la respuesta de la OMS a la COVID19. 2021.

Organización Mundial de la Salud. Nuevo coronavirus - República de Corea (procedente de China). Ginebra. Organización Mundial de la Salud. 2020.

Organización Mundial de la Salud. Nuevo coronavirus -Tailandia (procedente de China). Ginebra. Organización Mundial de la Salud. 2020.

$\mathrm{Wu} \mathrm{Z}$, McGoogan J. haracteristics of and important lessons from the coronavirus disease 2019 (COVID-19) outbreak in China: Summary of a report of 72314 cases from the Chinese Center for Disease Control and Prevention. JAMA. 2020. 